NOUVELLE

\section{Influence du climat sur la survenue des épidémies de méningite en Afrique de l'ouest}

Benjamin Sultan

IRD UR 086 LODYC/LOCEAN;

Laboratoire d'0céanographie

et de climat par

expérimentation et approche

numérique, UMR 7617

IRD-CNRS-UPMC; Université

Pierre et Marie Curie,

4 , place Jussieu,

75252 Paris Cedex 05,

France.

benjamin.sultan@

lodyc.jussieu.fr

> La méningite cérébro-spinale affecte l'Afrique sahélienne depuis des siècles, mais alors qu'avant les années 1980 elle touchait cette région de manière irrégulière, elle y est depuis devenue endémique. Dans les décennies 1980 et 1990 , l'Organisation mondiale de la santé (OMS) a enregistré entre 25000 et 200000 cas par an, dont environ $10 \%$ de cas mortels [1]. La méningite cérébrospinale est donc devenue un problème de santé publique particulièrement important dans une région parmi les plus pauvres du monde et déjà affectée par la diminution de la pluviométrie depuis la fin des années 1970 .

La méningite cérébro-spinale est une infection causée par la bactérie Neisseria meningitidis, très contagieuse et dont la transmission s'effectue par voie aérienne. Une combinaison de conditions favorables (relevant de l'environnement, de l'hôte et du microorganisme) est probablement nécessaire pour que survienne une épidémie [2]. La réceptivité immunologique de la population (peut-être due à la perte de l'immunité de groupe vis-à-vis de la souche prévalente), un niveau socio-économique bas, la transmission d'une souche virulente et un contexte climatique particulier (saison sèche, tempêtes de sable) ont été identifiés comme les conditions les plus importantes. Parmi ces conditions favorables, le facteur climatique joue un rôle non négligeable dans la chronologie de l'épidémie, qui démarre en février pour disparaître au mois de mai, et dans sa circonscription géographique dans la « ceinture de la méningite » située entre $10^{\circ} \mathrm{N}$ et $15^{\circ} \mathrm{N}$ $[1,3,4]$. En effet, la partie soudanosahélienne de l'Afrique est soumise à l'alternance d'une saison sèche en hiver, dominée par des vents secs du nord, l'Harmattan, et une saison humide de mousson en été. Le contexte climatique caractéristique de l'hiver des régions sahéliennes présente des conditions favorables au développement de la méningite: alors que la sécheresse et les vents forts, chargés de poussières, peuvent stimuler l'invasion du méningocoque en lésant directement la barrière muqueuse ou en inhibant les défenses immunitaires de surface, l'humidité du printemps et des latitudes guinéennes réduisent considérablement le risque de transmission [5]. Cependant, bien que I'influence globale du climat sur l'épidémie soit indiscutable, les effets de la variabilité climatique sur la dynamique épidémique sont encore mal connus du fait d'emboîtement de phénomènes d'échelles distinctes, d'interactions avec des facteurs biologiques (immunité, fragilité de l'organisme...), démographiques et socio-économiques.

Des travaux récents ont montré le rôle important joué par le climat sur la dynamique spatiale et temporelle de plusieurs maladies infectieuses $[6,7]$. Cependant, la mise en évidence de cet impact nécessite de faire la part entre I'hétérogénéité locale et le «forçage» à grande échelle dans la dynamique de la maladie [8]. L'agrégation des données locales est un moyen simple pour extraire la variabilité liée à la grande échelle tout en lissant l'hétérogénéité locale. C'est I'idée directrice de notre étude: montrer comment des phénomènes à grande échelle comme les modulations de l'Harmattan sur l'ensemble du Mali peuvent contribuer à la dynamique temporelle de l'épidémie à l'échelle nationale même si cette analyse agrégée au niveau national ne prend pas en compte des variations à des échelles plus fines. Cette étude repose sur des variables atmosphériques issues des ré-analyses produites par le National Center for Environmental Prediction et le National Center for Atmospheric Research [9], et sur des données épidémiologiques provenant de relevés hebdomadaires établis par l'OMS au Mali, entre 1994 et 2002. Des indices atmosphériques synthétiques ont été établis à partir d'une analyse en composante principale (ACP) sur la vitesse du vent en surface entre 1994 et 2002 ; ces indices ont ensuite été mis en parallèle avec les données de méningite cérébro-spinale au Mali. Pour chacune des neuf années, le maximum hivernal de l'Harmattan, défini en considérant la semaine où l'indice de vent est le plus élevé, correspond à la sixième semaine de l'année (entre le 7 et le 15 février). On observe un synchronisme entre l'évolution temporelle de l'indice atmosphérique et la progression de l'épidémie dont le démarrage coïncide avec la semaine du maximum hivernal de l'Harmattan (coefficient de corrélation: $0,92)$. Des résultats similaires ont été obtenus en utilisant les températures et 
I'humidité de surface pour la construction des indices atmosphériques [10].

Ces résultats, associant sciences du climat et de la santé, permettent d'envisager la mise en place d'outils de surveillance épidémiologique afin d'anticiper la survenue des épidémies de méningite dans cette région d'Afrique de l'ouest et tenter de limiter leur impact. Cependant, ces résultats ne permettent pas d'appréhender une éventuelle relation entre l'intensité de l'hiver et l'ampleur de l'épidémie. De plus, le modèle climatique utilisé se fondant sur une période de seulement neuf années, d'éventuelles variations importantes du climat ou des épidémies à plus grande échelle de temps ne peuvent pas être prises en compte. Ce modèle devra par conséquent être testé sur des périodes plus longues et à une échelle spatiale plus fine, afin d'étudier plus précisément ces variations et leur impact sur l'évolution de la maladie à l'intérieur du territoire malien. Le projet AMMA (analyse multidisciplinaire de la mousson africaine) et ses campagnes de mesures destinées à recueillir des mesures relatives, notamment au climat et à la santé en Afrique de l'ouest, devraient favoriser l'extension et la continuité de ces travaux dans une perspective de santé publique. $\diamond$

Influence of climate

upon the meningitis onset

in West Africa

\section{RÉFÉREWCES}

1. WHO. Report on global surveillance of epidemicprone infectious diseases 2000. Genève: OMS, 2001: $128 \mathrm{p}$.
2. WHO. Meningococcal meningitis. Fact Sheet 2003; 14l. http://www.who.int/mediacentre/factsheets/ 2003/fs14l/en/index.html

3. Lapeyssonnie L. La méningite cérébro-spinale en Afrique. Bull WHO 1963; 28 (suppl 1): 1-114.

4. Cheesbrough JS, Morse AP, Green SDR. Meningococcal meningitis and carriage in western Zaire: a hypoendemic zone related to climate? Epidemiol Infect 1995; 114: 75-92.

5. Chippaux JP, Chabalier F. Niger: une épidémie de méningite dévastatrice. Sciences Sud 2000; $6: 3$.

6. Rodo X, Pascual M, Fuchs G, Faruque AS. ENSO and cholera: a nonstationary link related to climate change? Proc Natl Acad Sci USA 2002; 99: 12901-6.

7. Pascual M, Rodo X, Ellner $S$, et al. Cholera dynamics and $\varepsilon$ I Nino-Southern oscillation. Science 2000; 289: 1766-9.

8. Guégan JF, Morand S, Poulin R. Are there general laws in parasite community ecology? The emergence of spatial parasitology and epidemiology. In: Thomas F, Renaud F, Guégan JF, eds. Parasitism and ecosystems. Oxford, UK: Oxford University Press, 2005 (sous presse).

9. Kalnay $\varepsilon$, Kanimatsu M, Kistler R, et al. The NCEP/NCAR 40-year reanalysis project. Bull Am Meteorol Soc 1996; 77 : 437-71.

10. Sultan B, Labadi K, Guégan JF, Janicot S. Climate drives the meningitis onset in West Africa. Plos-Med $2005 ; 2: \varepsilon 6$.

\section{NOUVELLE}

\section{Stimulation de la biosynthèse des protéoglycanes: une nouvelle stratégie pour la réparation du cartilage articulaire}

Narayanan Venkatesan, Jacques Magdalou, Patrick Netter, Sylvie Fournel-Gigleux, Mohamed Ouzzine

\author{
UMR CNRS 7561 - Université \\ Henri Poincaré, Nancy l, \\ Laboratoire de \\ Pharmacologie; \\ Faculté de Médecine, \\ BP 184, 54505 Vandœuvre- \\ les-Nancy Cedex, France. \\ ouzzine@
}

medecine.uhp-nancy.fr

tion, douleur), mais ces prescriptions conventionnelles ne permettent pas d'arrêter sa progression. De plus, l'utilisation de ces médicaments expose au risque de manifestations indésirables, voire toxiques, qui limitent leur utilisation chronique. Dans ce

> Les maladies articulaires telles que l'arthrose sont la conséquence de processus mécaniques et biologiques qui concourent à la rupture de l'homéostasie du cartilage et, à terme, à la dégradation de la fonction articulaire et de ses propriétés biomécaniques (Figure 1). Les arthropathies touchent plus de 900000 personnes dans le monde chaque année, et ont un coût important que l'on estime à plus de trois billions d'euros. Ces maladies, à la fois chroniques et évolutives, ont une prévalence élevée chez les sujets âgés. Elles altèrent considérablement la qua- lité de vie et sont une des principales causes de consultation médicale, de consommation médicamenteuse et d'invalidité.

II n'existe pas actuellement de traitement efficace des lésions dégénératives du cartilage. La mise en place de prothèses représente le stade ultime de traitement de l'arthrose et les approches pharmacologiques proposées sont limitées. Un éventail de substances pharmacologiques (anti-inflammatoires, antalgiques) est utilisé pour soulager les symptômes de la maladie (inflamma- contexte, les approches de bio-ingénierie tissulaire ouvrent des perspectives alternatives intéressantes dans le domaine de la réparation du cartilage, tissu incapable de se régénérer spontanément.

La perte d'homéostasie du cartilage est provoquée principalement par une production locale de cytokines pro-inflammatoires telles que l'interleukine-1 (IL-1), par les chondrocytes et les synoviocytes qui favorise à la fois la dégradation des constituants matriciels et l'inhibition de leur biosynthèse (Figure 1). 